\title{
Séance du Comité central du 23 août 2012
}

Groupe de travail «Art. 22a LAMal» - Suite aux réactions à l'article sur le projet MARS de l'Office fédéral de la statistique (OFS), le Comité central (CC) a décidé de réactiver le groupe de travail «Art. 22a LAMal». Celui-ci devra élaborer des contre-propositions aux démarches de l'OFS, préparer le dialogue avec le DFI et l'OFSP et planifier la suite des opérations.

Droit des brevets et liberté thérapeutique - Le Tribunal fédéral a admis la pratique de l'interdiction de la «deuxième indication thérapeutique». Un médicament ne peut ainsi être utilisé que pour l'indication figurant expressément dans l'information sur le produit. La FMH refuse cette restriction. Elle s'engage pour un meilleur «Gentlemen's Agreement» avec l'industrie pharmaceutique, et par la suite pour une modification de la loi.

Directives «Collaboration corps médical-industrie» - L'ASSM a révisé les directives «Collaboration corps médical-industrie». Hormis quelques modifications de moindre importance, la FMH approuve ces directives.
Projet TARVISION - La phase 1 du projet TARVISION (mise à jour de l'ensemble de la structure tarifaire) est quasiment terminée. La phase 2 sera consacrée en priorité au chapitre 40 portant sur les soins de premier recours. Dans le cadre de la revalorisation de la médecine de famille au moyen du Masterplan «Médecine de famille», la FMH traitera en premier le chapitre 40 avant de se consacrer aux autres sousprojets des sociétés de discipline médicale.

Forum Echange de données - Les normes pour les onze formulaires approuvés par l'organe spécialisé du Forum comprennent des informations dont les assureurs n'ont pas besoin et qui ne permettent plus de garantir l'anonymat des patients. La FMH s'engage pour l'introduction d'un numéro d'identification des patients qui, contrairement au numéro AVS, serait utilisé uniquement dans les hôpitaux.

Révision de la Déclaration d'Helsinki - L'Association médicale mondiale prévoit de réviser la Déclaration d'Helsinki, ce qui n'est pas impératif du point de vue de la FMH. Le Prof. Dominique Sprumont, Dr en droit, défendra la position de la FMH dans le groupe de travail de la WMA.

\section{Un registre pour vous trouver: doctorfmh.}

\author{
Etre là où les patients vous cherchent. \\ Gratuit pour nos membres.
}

